\title{
A saúde dos trabalhadores e a pandemia de COVID-19: da revisão à crítica
}

\section{The health of workers and COVID-19 pandemic: from review to criticism}

Diego de Oliveira Souza*

Universidade Federal de Alagoas, Arapiraca, AL, Brasil

\footnotetext{
* E-mail: diego.souza@arapiraca.ufal.br
}

\section{RESUMO}

Introdução: Em meio a um contexto atípico, com a emergência da pandemia de COVID-19, a ciência precisa dar respostas na mesma velocidade da propagação do SARS-CoV-2. A conjuntura tem sido especialmente violenta em relação aos trabalhadores dos serviços essenciais, sobremodo, os do setor saúde. Objetivo: Sintetizar, criticamente, as principais recomendações científicas para os trabalhadores ante a pandemia de COVID-19. Método: Trata-se de uma revisão de literatura a partir das bases PubMed e BVS, nas quais se obteve 19 publicações para análise. Resultados: Constatou-se ênfase para a questão dos EPI, medidas de higiene e outras medidas protetivas no âmbito individual. Alguns estudos abordam questões mais amplas, organizacionais e da esfera da vigilância voltada ao setor saúde, mas também aos trabalhadores em geral. Conclusões: Para além da síntese das publicações, a revisão contribui com reflexões e provocações com vistas às intervenções ulteriores.

PALAVRAS-CHAVE: Coronavírus; Pandemias; Saúde do Trabalhador; Trabalho

\begin{abstract}
Introduction: With the emergence of the COVID-19 pandemic, science needs to give quick answers. The context has been violent, especially for workers. Objective: critically summarize the main scientific recommendations for workers in the face of the COVID-19 pandemic. Method: This is a literature review from PubMed e VHL, in which 19 publications were obtained for analysis. Results: In the literature, emphasis was placed on the issue of PPE, hygiene measures and other individual measures. Some studies address organizational issues and surveillance for healthcare workers, but also for workers in general. Conclusions: In addition to the synthesis of publications, the review contributes with reflections and provocations with a view to further interventions.
\end{abstract}

KEYWORDS: Coronavirus; Pandemics; Occupational Health; Work 


\section{INTRODUÇÃO}

Este artigo possui o objetivo de revisar, criticamente, a literatura científica produzida sobre a saúde dos trabalhadores nos primeiros meses da pandemia de COVID-19, contribuindo para a identificação de recomendações para a prevenção da infecção e de suas consequências.

Em termo gerais, o estudo se justifica ante o caráter recente da pandemia, o que demanda esforços da comunidade científica no entendimento de seus múltiplos aspectos, subsidiando intervenções. Note-se que as autoridades sanitárias chinesas acionaram a Organização Mundial de Saúde (OMS), em 31 de dezembro de 2019, a respeito de casos de pneumonia na cidade de Wuhan (província de Hubei), na China, com suspeita de serem provocados por um novo tipo de coronavírus. Pouco mais de dois meses depois, em 11 de março, a situação é declarada, oficialmente, como uma pandemia da doença provocada pelo novo coronavírus, o SAR-CoV-21.

A disseminação global foi rápida, porquanto em 25 de abril de 2020 já eram 2.719.827 casos e 187.705 mortes no mundo. Os continentes mais afetados são o europeu e as Américas, porquanto na Europa, no mesmo dia, registravam-se 1.314 .666 casos e 119.463 mortes e, nas Américas, 1.047 .508 casos e 53.103 mortes, sendo 860.772 casos e 44.053 mortes só nos Estados Unidos da América (EUA)2.

Em termos específicos, a justificativa do estudo reside no fato de um dos principais problemas enfrentados durante a pandemia consistir no índice relevante de trabalhadores dos serviços essenciais infectados ou mortos por COVID-19, sobretudo aqueles do setor saúde. Até $1^{\circ}$ de abril de 2020 , por exemplo, eram cerca de 6.500 trabalhadores de saúde infectados na Espanha, 6.200 na Itália e 3.300 na China. Estima-se que, nos países mais afetados no primeiro trimestre de 2020 , de $4 \%$ a $12 \%$ dos casos confirmados sejam entre trabalhadores da saúde 3 .

Entre os aspectos que demandam maior preocupação, constam as condições de saúde e segurança dos trabalhadores que estão na linha de frente dos cuidados aos infectados, fator decisivo para o êxito do enfrentamento da pandemia. Além disso, outros impactos sobre os trabalhadores em geral já são percebidos, desde a própria infecção naqueles que atuam nos serviços que permaneceram funcionando durante o distanciamento social às questões psicoemocionais relativas ao medo pela infecção, perdas econômicas, mudança de rotina e isolamento'. Portanto, essa revisão buscou conhecer o que a ciência foi capaz de entender sobre a saúde dos trabalhadores nos primeiros quatros meses da pandemia.

\section{MÉTODO}

Trata-se de uma revisão de literatura que busca a abrangência dos estudos sobre a saúde dos trabalhadores durante a pandemia de COVID-19, integrando os conhecimentos já produzidos e submetendo-os a um diálogo crítico.
A busca de artigos foi realizada entre março e abril de 2020, nas seguintes bases de dados: National Library of Medicine (PubMed) e Biblioteca Virtual da Saúde (BVS). Tanto na PubMed quanto na BVS, a combinação de descritores utilizada foi: coronavirus and occupational health.

Foram selecionados textos publicados no período entre janeiro e abril de 2020, nos idiomas inglês, português e espanhol. Não foram incluídas publicações que abordavam doenças provocadas por outras cepas de coronavírus, que não se referiam à saúde dos trabalhadores ou duplicadas. Convém esclarecer que as publicações foram selecionadas independentemente do tipo de estudo, incluindo comunicações breves, editoriais, cartas e congêneres, dado o caráter recente dessa linha investigativa e a necessidade de abranger o máximo de conhecimento já produzido.

As publicações selecionadas foram lidas na íntegra, extraindo-se algumas de suas características: autores, revista, base de indexação, metodologia, idioma e síntese dos resultados/conclusões. Foi realizada uma síntese dos resultados e discussões das publicações para posterior reflexão crítica.

\section{RESULTADOS E DISCUSSÃO}

A busca na PubMed resultou em 15 publicações e, na BVS, em 13. No Quadro 1 foi demonstrado o detalhamento das duas buscas.

Comparando-se os resultados obtidos na PudMed e na BVS, identificou-se que dez publicações compareciam em ambas, seis apenas na PubMed e três apenas da BVS, totalizando 19 publicações, consoante a Figura.

Em relação às principais características das publicações, destaca-se que todas estão no idioma inglês, mas uma também possui versão em português, não houve repetição de revistas, sendo, portanto, 19 periódicos distintos. A maior parte das publicações foi de caráter sintético, como comunicações breves, cartas ou comentários $(n=14)$, mas também houve uma revisão de

Quadro 1. Seleção dos artigos na PubMed e na BVS (jan-abr 2020).

\begin{tabular}{|lcc|}
\hline & PubMed & BVS \\
\hline $\begin{array}{l}\text { Resultado da busca (coronavirus and } \\
\text { occupational health) }\end{array}$ & 121 & 86 \\
$\begin{array}{l}\text { Seleção dos textos publicados em 2020 } \\
\text { Seleção dos textos publicados em periódicos }\end{array}$ & 22 & 24 \\
$\begin{array}{l}\text { Seleção dos textos escritos em inglês, português } \\
\text { ou espanhol }\end{array}$ & 22 & 18 \\
\hline Total parcial da seleção & 22 & 18 \\
\hline $\begin{array}{l}\text { Exclusão dos textos que abordavam outros vírus } \\
\text { ou doenças }\end{array}$ & 4 & 3 \\
$\begin{array}{l}\text { Exclusão dos textos que não se referiam à saúde } \\
\text { dos trabalhadores }\end{array}$ & 2 & 0 \\
$\begin{array}{l}\text { Exclusão por repetição dentro da mesma base } \\
\text { Total em cada busca após exclusões }\end{array}$ & 0 & 2 \\
\hline
\end{tabular}

Fonte: Elaborado pelo autor, 2020.

PubMed: National Library of Medicine; BVS: Biblioteca Virtual da Saúde. 


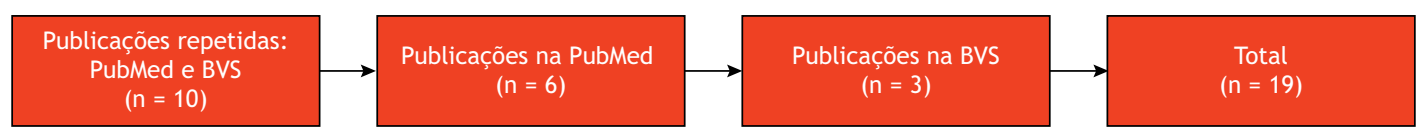

Fonte: Elaborada pelo autor, 2020.

Figura. Distribuição das publicações por busca e base de indexação (jan-abr 2020).

literatura, duas revisões sistemáticas e um ensaio clínico. No Quadro 2, apresenta-se o panorama das características extraídas das publicações na revisão.

Quanto aos resultados e discussões, constatou-se, predominantemente, abordagens voltadas aos trabalhadores de saúde. Entre esses, houve aqueles que se dedicaram a ramos especializados ou às medidas organizacionais e coletivas dentro das instituições de saúde. Por fim, ainda houve aqueles que abordaram a saúde dos trabalhadores em geral.

Riscos e recomendações aos trabalhadores da saúde

Na busca por evidências sobre a distância segura entre indivíduos, Bahl et al. ${ }^{4}$ constataram que a recomendação de 1 a 2 metros não é segura em todas as situações, pois há provas de que algumas gotículas alcançam até 8 metros. Os estudos disponíveis mostram também que o SARS-CoV-2 pode ser detectado no ar $3 \mathrm{~h}$ após a pulverização. Recomendam precaução e adoção de proteção pessoal de alto nível aos trabalhadores de saúde, mesmo mantendo distância na maior parte do tempo.

A questão do uso de equipamentos de proteção individual (EPI) é o foco da abordagem de Heinzerling et al. ${ }^{5}$. Os autores constataram três trabalhadores infectados entre 43 testados após contato com paciente de COVID-19. Dois deles executavam procedimentos de alto risco e desenvolveram os sintomas. Ambos relataram que passaram cerca de $3 \mathrm{~h}$ junto ao paciente durante assistência respiratória. Nenhum dos dois usou, de forma permanente, uma máscara facial, respirador, proteção dos olhos ou bata. 0 terceiro infectado pelo vírus, que se encontrava em risco médio, relatou contato próximo com o doente durante um total de $2 \mathrm{~h}$, mas não realizou procedimentos com aerossóis. Este trabalhador referiu usar EPI, mas removia ocasionalmente a máscara para falar e não usava óculos de proteção.

O uso do EPI se mostra, de fato, decisivo para a prevenção da COVID-19 entre trabalhadores de saúde, mas desde que seja de material adequado e utilizado da maneira correta. Verbeek et al. ${ }^{6}$ demonstraram, em estudos de simulação para colocação e retirada dos EPI, que as taxas médias de contaminação foram de $25 \%$ para os grupos que receberam treinamento e de $67 \%$ para os grupos de controle.

A qualidade do equipamento é da maior importância, uma vez que modificações no design do EPI podem diminuir a contaminação, como por exemplo: bata selada e com colarinho ajustável

Quadro 2. Principais características das publicações revisadas (jan-abr 2020).

\begin{tabular}{|c|c|c|c|c|}
\hline Autores & Revista & Base de dados & Idioma & Tipo de estudo \\
\hline Bahl et al. ${ }^{4}$ & The Journal of Infectious Diseases & PubMed/BVS & Inglês & Revisão sistemática \\
\hline Heinzerling et al. ${ }^{5}$ & Morbidity and Mortality Weekly Report & PubMed/BVS & Inglês & Relatório \\
\hline Verbeek et al. ${ }^{6}$ & Cochrane Database of Systematic Reviews & PubMed & Inglês & Revisão sistemática \\
\hline Antal et al. ${ }^{7}$ & Orvosi Hetilap & PubMed & Inglês & Revisão de literatura \\
\hline Spinazzè et al. ${ }^{8}$ & Annals of Work Exposures and Health & PubMed/BVS & Inglês & Comentário \\
\hline Zhang et al. ${ }^{9}$ & Frontiers of Medicine & PubMed/BVS & Inglês & Comunicação breve \\
\hline Elston $^{10}$ & Journal of the American Academy of Dermatology & PubMed/BVS & Inglês & Carta ao editor \\
\hline Zhao et al. ${ }^{11}$ & Journal of Cardiothoracic and Vascular Anesthesia & PubMed/BVS & Inglês & Ensaio clínico \\
\hline Gallasch et al. ${ }^{12}$ & Rev Enferm UERJ & BVS & Inglês/Português & Artigo de atualidade \\
\hline $\mathrm{Li}^{13}$ & Emerging Microbes \& Infections & BVS & Inglês & Protocolo \\
\hline Gan et al. ${ }^{14}$ & Safety and Health at Work & PubMed/BVS & Inglês & Comunicação breve \\
\hline Bann et al. ${ }^{15}$ & Head \& Neck & PubMed/BVS & Inglês & Comunicação breve \\
\hline Chen, Chi $^{16}$ & Cancer Cytopathology & PubMed & Inglês & Comentário \\
\hline Carver, Phillips ${ }^{17}$ & Workplace Health \& Safety & PubMed/BVS & Inglês & Comunicação breve \\
\hline Gudi, Tiwari ${ }^{18}$ & $\begin{array}{c}\text { International Journal of Occupational and } \\
\text { Environmental Medicine }\end{array}$ & PubMed & Inglês & Comunicação breve \\
\hline Koh $^{19}$ & Occupational Medicine & PubMed/BVS & Inglês & Editorial \\
\hline $\operatorname{Sim}^{20}$ & Occupational and Environmental Medicine & PubMed & Inglês & Editorial \\
\hline Fadel et al. ${ }^{21}$ & The Lancet. Public Health & PubMed & Inglês & Carta \\
\hline Liem et al. ${ }^{22}$ & The Lancet. Psychiatry & BVS & Inglês & Carta \\
\hline
\end{tabular}

Fonte: Elaborado pelo autor, 2020.

PubMed: National Library of Medicine; BVS: Biblioteca Virtual da Saúde. 
ao pescoço, pulsos e mãos; e a melhor cobertura da interface bata/punho ${ }^{6}$. Além do uso correto, a guarda e a substituição no tempo adequado são fatores a serem considerados ${ }^{7}$.

A alta demanda, repentina, por EPI tem ocasionado a falta desses suprimentos, o que gera angústia e outras cargas psíquicas para os trabalhadores da linha de frente ${ }^{19}$, bem como discussões polêmicas por parte dos gestores, a respeito de reutilização e gestão de reposição. Sobre isso, Spinazzè et al. ${ }^{8}$ trouxeram as recomendações da Associazione Italiana degli Igienisti Industriali (AIDII), quando destacam que deve haver uso racional de EPI, não recomendando a reutilização, uma vez que, em geral, não há comprovação de sua eficácia após desinfecção, pois ocorrem prováveis modificações do material. Fizeram a ressalva de que, havendo comprovação da eficácia, a reutilização pode ser uma medida para situações excepcionais, de emergência, mas nunca rotina. Por conta disso, Zhang et al. ${ }^{9}$ defenderam a criação de sistemas centralizados e redes coordenadas de fornecimento rápido de EPI, com articulação das várias esferas governamentais, unidades e equipes de saúde.

Outra face da questão dos EPI é o seu uso prolongado e as sequelas decorrentes. Elston ${ }^{10}$ relatou a ocorrência de danos à saúde pelo uso prolongando, como lesões cutâneas no nariz, mãos, bochechas e testa, destacou que os óculos se mostram mais frequentemente associados a lesões do que as máscaras e recomendou EPI com ajustes anatômicos mais adequados, adoção de turnos de plantão mais curtos, testagem para sensibilidade cutânea e uso de películas de barreiras e luvas sem látex.

Outras medidas no âmbito da proteção dos trabalhadores de saúde passam pela higienização das mãos ${ }^{10,11,12}$, monitoração de sinais e sintomas e diagnóstico precoce ${ }^{9,13,12}$, afastamento dos indivíduos do grupo de risco das atividades clínica e laboratoriais $^{13}$, desinfecção nos hotéis ou acomodações que os trabalhadores se instalam e formação de equipe de especialistas para atender outros trabalhadores do setor saúde?.

Medidas de alcance organizacional e coletivo nas unidades de saúde

A questão do ambiente e processos pode favorecer ou dificultar a propagação do SARS-CoV2, o que confere relevância às medidas de engenharia e gestão nas instituições de saúde. Nesse contexto, Gan et al. ${ }^{14}$ propuseram um modelo de engenharia de sistemas para prevenção da infecção intra-hospitalar. No modelo, o elemento central é o trabalhador da saúde e, nos vértices, estão as tarefas de trabalho, as tecnologias e ferramentas, os fatores ambientais e as condições organizacionais. Algumas medidas sugeridas foram: segregação daqueles que cuidam dos indivíduos suspeitos ou confirmados com COVID-19; estratificação das tarefas com vistas a escolha dos níveis de EPI; testagem e monitoração de temperatura dos trabalhadores da saúde; apoio e orientações contínuas; monitoração dos sintomas e restrição de visitantes.

A utilização de engenharia e de medidas destinadas a favorecer a ventilação adequada das salas pode contribuir para a redução das chances de infecção ${ }^{8}$. Realização de procedimentos cirúrgicos em sala com pressão negativa ${ }^{11}$; medidas que impeçam aglomerações no pré-atendimento ${ }^{12}$, restrição de visitantes nas instituições ${ }^{14}$, definição de medidas processuais e organizacionais gerais ${ }^{8}$ ou específicas para cada nível de atençãa ${ }^{5,15,16}$ foram algumas das medidas de alcance mais amplo encontradas na literatura. Os treinamentos específicos para quem está na linha de frente ${ }^{6,9,13}$, bem como o estabelecimento de redes de vigilância epidemiológica específica para os trabalhadores da saúde ${ }^{9,12}$ também são citadas como importantes estratégias de caráter coletivo.

Apesar de o destaque ficar por conta da proteção pessoal, essas medidas de maior abrangência são fundamentais para a mitigação da disseminação da COVID-19 nos serviços de saúde.

\section{Recomendações para trabalhadores de saúde especializados}

Três artigos discutem situações específicas de serviços especializados de saúde. Além das medidas básicas de higiene, EPI básicos e distanciamento, apresentam protocolos para situações específicas.

Bann et al. ${ }^{15}$ recomendaram medidas protetivas para os otorrinolaringologistas, tais como o adiamento de procedimentos eletivos e, naqueles que não possam ser adiados, manter equipe mínima à realização; testagem de todos da equipe $48 \mathrm{~h}$ antes do procedimento; não utilização de cânula nasal de alto fluxo nos cuidados em pacientes com traqueostomia e uso de EPI de alta proteção nesses casos; utilização de laringoscópio de vídeo e descartável nas intubações; realização de teste para SARS-CoV-2 $48 \mathrm{~h}$ antes em pacientes que requeiram cirurgias de urgência nas vias aéreas superiores, seguida de quarentena $e$ realização de teste rápido no pré-operatório imediato; e atendimento por telessaúde para os casos clínicos.

Para o caso dos procedimentos de anestesia, Zhao et al. ${ }^{11}$ elaboraram um protocolo que inclui triagem antes da admissão dos pacientes para detecção de casos suspeitos ou confirmados. Nesses casos, o indivíduo deve ser encaminhado para sala de cirurgia de pressão negativa, com medidas de alta proteção para os trabalhadores da saúde, o que inclui os EPI. Após o procedimento, deve-se realizar a completa desinfecção e esterilização da sala e a equipe atuante deve realizar higienização rigorosa de corpo todo.

Chen e $\mathrm{Chi}^{16}$ recomendaram medidas de biossegurança em laboratórios de citopatologia, estratificando-as por tipo de procedimento. Em geral, sugeriu-se a avaliação e o controle dos riscos em cada tipo de ensaio; formação sobre prevenção e controle de infecções; disponibilização de EPI adequado ao nível de risco de cada estrato das atividades; acesso a serviços de aconseIhamento e apoio psicológico; garantia de proteção ambiental; desinfecção rigorosa de superfícies; incentivo à comunicação de acidentes, autoavaliação e comunicação dos sintomas; adaptação das jornadas de trabalho e aumento das pausas; garantia de transporte seguro; garantia de guarda e transporte adequado do material coletado; ambientação adequada para o nível de atividade; diminuição da produção de aerossóis nos procedimentos; eliminação adequada de todos os EPI e resíduos; e limitação do número de pessoas presentes nas salas de procedimentos. 
Otorrinolaringologistas, anestesiologistas e trabalhadores de laboratório, entre outros, são grupos que estão sob o mais alto grau de risco biológico, porquanto haja contato direto com aerossóis, secreções ou coleta de material para diagnóstico. Faz-se mister a constante avaliação e atualização dos resultados das medidas implementadas para proteção desses grupos.

\section{Riscos e recomendações para os trabalhadores em geral}

Seis publicações dão destaque para a COVID-19 como doença relacionado ao trabalho fora do setor saúde. Carver e Phillips ${ }^{17}$ mencionaram medidas a serem adotadas por parte dos empregadores, especialmente o estabelecimento de home office para os trabalhadores e quarentena para os sintomáticos. Destacaram o papel dos enfermeiros do trabalho na educação dos trabalhadores na adoção de medidas preventivas de higiene e esclarecimentos sobre o afastamento social. Gudi e Tiwari ${ }^{18}$ corroboraram, destacando a importância da adoção do trabalho remoto desde casa e o rodízio de equipes no local de trabalho.

Para Koh ${ }^{19}$, a COVID-19 é a primeira nova doença profissional dessa década, o que demanda a rápida detecção das ocupações de maior exposição. Segundo o autor, além dos trabalhadores da saúde, os últimos fatos revelaram que merecem atenção: vendedores de artigos hospitalares, trabalhadores domésticos, guias turísticos, ourives que atendem turistas, executivos de multinacionais, taxistas, motoristas particulares, agentes de segurança, funcionários de cassinos e resorts e trabalhadores da construção. $\mathrm{Sim}^{20}$ acrescentou outras ocupações, como tripulações marítimas e aéreas, pessoal dos serviços de emergência (policiais, bombeiros etc.), cuidadores, educadores, trabalhadores domésticos, da alimentação e os motoristas de transportes públicos.

Para preservar a saúde desses trabalhadores, além das medidas de teletrabalho e quarentena, foram reforçadas as medidas de higiene, assim como foi destacada a importância do rastreamento, notificação e vigilância da doença, ações educativas e acompanhamento por telessaúde ${ }^{21}$. No caso dos trabalhadores imigrantes, o smartphone foi citado como um aliado no acesso às informações em idioma de origem, dirimindo o desfavorecimento com o qual se deparam nos sistemas de saúde dos países onde passaram a viver ${ }^{22}$.

Dado o caráter recente da pandemia, muitas mediações da relação entre COVID-19 e trabalho devem ser, ainda, problematizadas. Por ora, urge a necessidade de disseminar e implantar medidas que protejam os trabalhadores e aliviem a carga dos sistemas de saúde no mundo.

\section{Da revisão à crítica}

A questão mais evidente na revisão diz respeito à recomendação sobre os EPI, o que é compreensível ante a necessidade mais urgente de interromper a transmissão. Na mesma esteira, destacam-se as medidas de higiene e desinfecção, sobretudo para os trabalhadores da saúde, expostos a altos níveis de riscos biológicos, quando essas medidas individuais são decisivas para a preservação da saúde.

As dificuldades para a proteção de trabalhadores de saúde, das quais agora se evidencia a falta de EPI, é questão crônica em vários países, antecedendo à pandemia. A fase atual exponencia e evidencia o problema para o mundo, o que deve demandar reflexões no sentido de pensar o que a ele subjaz. Aqui, considerando a ampliação da abordagem crítica, destaca-se a articulação que esse problema apresenta com um contexto de fragilidade de sistemas de saúde pública, muitas vezes subfinanciados, com déficit no quantitativo de trabalhadores, leitos e recursos estruturais em geral. Equacionar essa questão na base dos sistemas de saúde é peremptório para um horizonte de maior consistência no enfrentamento de situações emergenciais de saúde pública, mas também das cotidianas ${ }^{23}$.

Nessa mesma perspectiva, a esfera da Vigilância em Saúde do Trabalhador (VISAT) deve ser fortalecida, a fim de gerar informações precisas e, portanto, práticas efetivas e eficientes. Convém destacar que a elaboração/execução das ações de VISAT pode se dar como um mero procedimento burocrático e institucional, no qual o trabalhador é objeto vigiado, ou, em outra perspectiva, considerando que

[...] existe uma variável transversal que sustenta a própria existência da VISAT, sua razão de ser: o trabalhador, com seu saber próprio, representado socialmente da forma mais adequada na conjuntura em que se insere (sindicato, associação, movimento, comissão, conselho etc.). Não há VISAT sem o protagonismo daquele que é o anteparo mais imediato dos impactos que os processos produtivos causam à sua saúde ${ }^{24}$.

A segunda perspectiva mencionada é a que alcança a amplitude pretendida para a VISAT, devendo ser a matriz teórico-metodológica a fundamentar as recomendações, políticas, programas e protocolos que envolvam a VISAT, quando o saber dos próprios trabalhadores possui função estruturante.

Vinculada a essa ideia, comparece a perspectiva de fortalecimento das atividades educativas, que na produção revisada estiveram orientadas à prevenção da infecção em geral ou à expertise profissional para atuação nos serviços de saúde. Essas ações também devem ser pensadas e executadas com protagonismo dos trabalhadores, enquanto sujeitos ativos, no sentido de que a autoridade científica dos técnicos não se sobreponha ou ignore a autoridade do saber dos trabalhadores sobre as relações estabelecidas no processo de trabalho ${ }^{25}$. Obviamente, atividades educativas dessa amplitude se revestem de complexidade e tensões para com o status quo, pressupõem organizações coletivas consistentes, o que só faz ao longo de um processo histórico.

A defasagem nesse processo não pode ser sanada, em sua plenitude, em meio ao "calor" de uma emergência de saúde pública, o que faz imperar, por vezes, o pragmatismo. Nesse contexto, com o problema já em curso, são bem-vindas as atividades de educação das várias perspectivas que visem contribuir para o enfrentamento da pandemia, com a ressalva de que o horizonte precisa ser mais amplo, com o fortalecimento da VISAT, da educação em saúde e da educação permanente, orientadas pela perspectiva dos trabalhadores com vistas à antecipação dos problemas. 
Esse horizonte é válido para o conjunto dos trabalhadores, uma vez que, apesar de eles estarem expostos ao SARS-CoV-2 em um nível menor do que os trabalhadores do setor saúde, eles estão sob cargas de trabalho de natureza diversa, sobretudo em meio às mudanças sociais corolárias à pandemia. Chama-se a atenção às implicações econômico-sociais e psicoemocionais relativas ao isolamento social, mas com a ressalva de que, na perspectiva crítica aqui defendida, não é a pandemia a responsável pela fragilização social a que estão submetidos (embora seja um fator agravante), mas, ao contrário, é um sistema econômico-social desigual que antecede à pandemia que os coloca em situação desfavorecida em face do necessário isolamento. Destaca-se que em uma publicação ${ }^{22}$ foi realizada alusão às condições desiguais pregressas que reverberam no contexto da pandemia, quando se cita o caso dos trabalhadores que são imigrantes em várias nações e se veem desprotegidos em uma situação duplamente atípica: a pandemia e o estar no espaço alheio.

Essa reflexão deve se estender no sentido da apreensão das diferenças existentes entre as nações e, no interior delas, entre os estratos da classe trabalhadora. Ademais, considerar a estrutura desigual que emerge das relações de produção é decisivo para o entendimento de que parte (considerável) dos trabalhadores já vive, cotidianamente, em uma situação socialmente limítrofe, quando a saúde (ou a perda dela) é, também, via de expressão dessa desigualdade ${ }^{26}$. Esse pressuposto convém à análise, inclusive, dos trabalhadores da saúde, imersos em um contexto de precarização do trabalho e das instituições nas quais atuam, o que eclodiu com violência face à pandemia.

Com efeito, além da pecha heroica ora atribuída aos trabalhadores da saúde e outros serviços essenciais, eles precisam de melhores condições de trabalho; melhor qualificação; sistemas de saúde mais bem estruturados; políticas, programas e protocolos mais amplos que permitam o exercício do protagonismo que lhes é de direito e, enfim, a transformação da dinâmica de trabalho que os avilta diariamente. Para além da questão dos EPI e medidas de higiene (importantíssimas), faz-se mister mudanças mais radicais, isto é, desde as raízes. Enfrentar uma problemática da proporção de uma pandemia tendo o peso desse processo histórico, sem dúvida, tem sido um entrave ao êxito pretendido, o que só retroalimenta a carga que se volta, fatidicamente, contra os próprios trabalhadores.

\section{CONCLUSÕES}

A revisão de literatura realizada neste artigo permitiu perceber que a ciência tem dado respostas rápidas à COVID-19, quando já há um razoável número de publicações em poucos meses. No caso específico da saúde dos trabalhadores, conseguiu-se, aqui, revisar 19 publicações que apresentam recomendações, reflexões e evidências decisivas para o enfrentamento da pandemia, especialmente visualizando o universo dos trabalhadores da saúde, mas também com contribuições para os trabalhadores em geral.

Além de sintetizar os resultados e discussões dessas publicações, o artigo ora apresentado contribuiu para, a partir e para além da revisão, trazer pontos para a reflexão crítica e algumas provocações. Certamente, ainda há um longo caminho a ser percorrido pela ciência e pela filosofia a fim de entender os múltiplos aspectos da pandemia que repercutem na saúde dos trabalhadores e, assim, subsidiar intervenções ulteriores.

\section{REFERÊNCIAS}

1. Representação da Organização Pan-americana da Saúde no Brasil - OPAS Brasil. COVID-19 (doença causada pelo novo coronavírus). Folha Informativa. 2020[acesso 26 abr 2020]. Disponível em: https: / /www.paho.org/bra/index.php?option=com_ content\&view $=$ article\&td $=6101$ : covid $19 \& \mid$ temid $=875$

2. World Health Organization - WHO. Coronavirus disease 2019 (COVID-19): situation report 96. Genebra: World Health Organization; 2020[acesso 26 abr 2020]. Disponivel em: https://www.who.int/docs/defaultsource/coronaviruse/situation-reports/20200425-sitrep96-COVID-19.pdf?sfvrsn=a33836bb_2\&ua=1

3. British Broadcast Corporation - BBC. Coronavírus: por que a COVID-19 afeta tanto os profissionais de saúde? BBC News Brasil. 1 abr 2020[acesso 27 abr 2020]. Disponível em: https://www.bbc.com/portuguese/internacional-52119508

4. Bahl P, Doolan C, Silva C, Chughtai AA, Bourouiba L, MacIntyre CR. Airborne or droplet precautions for health workers treating COVID-19? J Infect Dis. 2020:1-29. https://doi.org/10.1093/infdis/jiaa189

5. Heinzerling A, Stuckey JM, Scheuer T, Xu K, Perkins KM, Resseger $\mathrm{H}$ et al. Transmission of COVID-19 to health care personnel during exposures to a hospitalized patient: Solano county, California, february 2020. MMWR Morb Mortal Wkly Rep. 2020;69(15):472-6. https://doi.org/10.15585/mmwr.mm6915e5

6. Verbeek JH, Rajamaki B, Ijaz S, Sauni R, Toomey E, Blackwood B et al. Personal protective equipment for preventing highly infectious diseases due to exposure to contaminated body fluids in healthcare staff. Cochrane Database Syst Rev. 2020;4(4):1-147. https://doi.org/10.1002/14651858.CD011621.pub4

7. Antal M, Szabó RM, Juhász Z, Vereb T, Piffkó J. New information for the clinical detection of COVID-19 virus infection and options for protection of healthcare workers in the head and neck region. Orv Hetil. 2020;161(17):660-6. https: / / doi.org/10.1556/650.2020.31806

8. Spinazzè A, Cattaneo A, Cavallo DM. COVID-19 outbreak in Italy: protecting worker health and the response of the italian industrial. Ann Work Expo Health. 2020:1-6. https://doi.org/10.1093/annweh/wxaa044

9. Zhang Z, Liu S, Xiang M, Li S, Zhao D, Huang C et al. Protecting healthcare personnel from 2019-nCoV infection risks: lessons and suggestions. Front Med. 2020;14(2):229-31. https://doi.org/10.1007/s11684-020-0765-x 
10. Elston DM. Occupational skin desease amon health care workers during the coronavírus (COVID-19) epidemic. J Am Acad Dermatol. 2020;82(5):1085-6. https://doi.org/10.1016/j.jaad.2020.03.012

11. Zhao $S$, Ling $K$, Yan $H$, Zhong L, Peng $X$, Yao $S$ et al. Anesthetic management of patients with COVID-19 infections during emergency procedures. J Cardio Vasc Anest. 2020;34(5):1125-31. https://doi.org/10.1053/j.jvca.2020.02.039

12. Gallasch CH, Cunha ML, Pereira LAS, Silva Junior JS. Prevenção relacionada à exposição ocupacional: COVID-19. Rev Enferm UERJ. 2020;28:1-6. https://doi.org/10.12957/reuerj.2020.49596

13. Li T. Diagnosis and clinical management of severe acute respiratory syndrome coronavirus 2 (SARS-CoV-2) infection: an operational recommendation of peking union medical college hospital (V2.0). Emerg Microbes Infect. 2020;9(1):582-5. https://doi.org/10.1080/22221751.2020.1735265

14. Gan HW, Lim JW, Koh D. Preventing intra-hospital infection and transmission of coronavirus disease 2019 in healthcare workers. Saf Health Work. 2020;1-3. https://doi.org/10.1016/j.shaw.2020.03.001

15. Bann DV, Patel VA, Saadi R, Gniady JP, Goyal N, McGinn JD et al. Impact of coronavirus (COVID-19) on otolaryngologic surgery: brief commentary. Head Neck. 2020;42(2):1227-34. https://doi.org/10.1002/hed.26162

16. Chen CC, Chi CY. Biosafety in the preparation and processing of cytology specimens with potential coronavirus (COVID-19) infection: perspectives from Taiwan. Cancer Cytopathol. 2020;108(5):309-16. https://doi.org/10.1002/cncy.22280

17. Carver PE, Phillips J. Novel coronavirus (COVID-19): what you need to know. Workplace Health Saf. 2020;68(5):250. https: //doi.org/10.1177/2165079920914947
18. Gudi SK, Tiwari KK. Preparedness and lessons learned from the novel coronavirus disease. Int J Occup Environ Med. 2020;11(2):108-12. https://doi.org/10.34172/ijoem.2020.1977

19. Koh D. Occupational risks for COVID-19 infection. Occup Med (Lond). 2020;12(70):3-5. https://doi.org/10.1093/occmed/kqaa036

20. Sim MR. The COVID-19 pandemic: major risks to healthcare and other workers on the front line. Occup Environ Med. 2020;77(5):281-2. https://doi.org/10.1136/oemed-2020-106567

21. Fadel M, Salomon J, Descatha A. Coronavirus outbreak: the role of companies in preparedness and responses. Lancet Public Health. 2020;5(4):1. https://doi.org/10.1016/S2468-2667(20)30051-7

22. Liem A, Wang C, Wariyanti Y, Latkin CA. The neglected health of international migrant workers in the COVID-19 epidemic. Lancet Psychiatry. 2020;7(4):20. https://doi.org/10.1016/S2215-0366(20)30076-6

23. Souza DO. Financeirização, fundo público e os limites à universalidade da saúde. Saude Deb. 2019;43(esp. 5):71-81.

24. Vasconcellos LCF. Vigilância em saúde do trabalhador: decálogo para uma tomada de posição. Rev Bras Saude Ocup. 2018;43(supl. 1):1-9. https://doi.org/10.1590/2317-6369000029517

25. Vasconcellos LCF, Almeida CVB, Guedes DT. Vigilância em saúde do trabalhador: passos para uma pedagogia. Trab Educ Saude. 2009;7(3):445-62. https://doi.org/10.1590/S1981-77462009000300004

26. Souza DO, Melo AISC, Vasconcellos LCF. A saúde dos trabalhadores em "questão": anotações para uma abordagem histórico-ontológica. Soc Quest. 2015;18(34):107-36.

Contribuição dos Autores

Souza DO - Concepção, planejamento (desenho do estudo), aquisição, análise, interpretação dos dados e redação do trabalho. 0 autor aprovou a versão final do trabalho.

Conflito de Interesse

Os autores informam não haver qualquer potencial conflito de interesse com pares e instituições, políticos ou financeiros, deste estudo.

Esta publicação está sob a licença Creative Commons Atribuição 3.0 não Adaptada.

Para ver uma cópia desta licença, visite http://creativecommons.org/licenses/by/3.0/deed.pt_BR. 